\title{
SUPER RESOLUTION OF MULTISPECTRAL IMAGES USING $\ell 1$ IMAGE MODELS AND INTERBAND CORRELATIONS
}

\author{
Miguel Vega ${ }^{a}$, Javier Mateos $^{b}$, Rafael Molina ${ }^{b}$, and Aggelos K. Katsaggelos $^{c}$ \\ a) Dept. de Lenguajes y Sistemas Informáticos, Univ. de Granada, 18071 Granada, Spain \\ b) Dept. de Ciencias de la Computación e I. A., Univ. de Granada, 18071 Granada, Spain \\ c) Dept. of Electrical Engineering and Comp. Sci., Northwestern Univ., Evanston, Illinois 60208-3118 \\ mvega@ugr.es,jmd@decsai.ugr.es,rms@decsai.ugr.es, aggk@eecs.northwestern.edu
}

\begin{abstract}
In this paper we propose a novel super-resolution based algorithm for the pansharpening of multispectral images. Within the Bayesian formulation, the proposed methodology incorporates prior knowledge on the expected characteristics of multispectral images; that is, imposes smoothness within each band by means of the energy associated to the $\ell 1$ norm of vertical and horizontal first order differences of image pixel values and also takes into account the correlation between the bands of the multispectral image. The observation process is modeled using the sensor characteristics of both panchromatic and multispectral images. The method is tested on real and synthetic images, compared with other pansharpening methods, and its quality is assessed both qualitatively and quantitatively.
\end{abstract}

\section{INTRODUCTION}

Nowadays most remote sensing systems include sensors able to capture, simultaneously, several low resolution images of the same area on different wavelengths, forming a multispectral image, along with a high resolution panchromatic image. The main advantage of the multispectral image is to allow for a better land type and use recognition but, due to its lower resolution, information on the objects shape and texture may be lost. In contrast, the panchromatic image allows for a better recognition of the objects in the image and their textures but provides no information about their spectral properties.

The super resolution of multispectral images, also called pansharpening, is a technique that jointly processes the multispectral and panchromatic images in order to obtain a new multispectral image that, ideally, exhibits the spectral characteristics of the observed multispectral image and the resolution of the panchromatic image. A few approaches to pansharpening have been proposed in the literature, for instance, $[1,2,3]$ and a comparison of algorithms in [4]. In this paper we propose the use, within the Bayesian formulation, of an image model which imposes smoothness into each band of the pansharpened multispectral image by mean of the $\ell 1$ norm of vertical and horizontal first order differences of image pixel values and takes into account the correlation between the bands of the multispectral image. To the best of our knowledge,

This work has been supported by the "Comisión Nacional de Ciencia y Tecnología" under contract TIN2007-65533 and the Consejería de Innovación, Ciencia y Empresa of the Junta de Andalucía under contract P07-TIC-02698. this correlation is the first time that it is explicitly incorporated into the modeling of the pansharpening problem.

The paper is organized as follows. In section 2 the Bayesian modeling and inference for super resolution reconstruction of multispectral images is presented. Section 3 describes the variational approximation of the posterior distribution of the high resolution multispectral image and how inference is performed. Section 4 presents experimental results and section 5 concludes the paper.

\section{BAYESIAN MODELING AND INFERENCE}

Let us assume that $\mathbf{y}$, the unknown high resolution multispectral image we would have observed under ideal conditions, has $B$ bands $\mathbf{y}_{b}, b=1, \ldots, B$, each of size $p=m \times n$, that is, $\mathbf{y}=\left[\mathbf{y}_{1}^{t}, \mathbf{y}_{2}^{t}, \ldots, \mathbf{y}_{B}^{t}\right]^{t}$, where each band of this image is expressed as a column vector by lexicographically ordering the pixels in the band, and $t$ denotes the transpose of a vector or matrix. The observed low resolution multispectral image $\mathbf{Y}$ has $B$ bands $\mathbf{Y}_{b}, b=1, \ldots, B$, each of size $P=M \times N$ pixels, with $M<m$ and $N<n$. These images are also stacked into the vector $\mathbf{Y}=\left[\mathbf{Y}_{1}^{t}, \mathbf{Y}_{2}^{t}, \ldots, \mathbf{Y}_{B}^{t}\right]^{t}$, where each band of this image is also expressed as a column vector by lexicographically ordering the pixels in the band. The sensor also provides us with a panchromatic image $\mathbf{x}$ of size $p=m \times n$, obtained by spectrally averaging the high resolution images $\mathbf{y}_{b}$.

The objective of the super resolution of multispectral images is to obtain an estimate of the unknown high resolution multispectral image $\mathbf{y}$ given the panchromatic high resolution observation $\mathbf{x}$ and the low resolution multispectral observation $\mathbf{Y}$. The Bayesian formulation of this problem requires the definition of the joint distribution $\mathrm{p}(\mathbf{y}, \mathbf{Y}, \mathbf{x})$. We define this joint distribution as $\mathrm{p}(\mathbf{y}, \mathbf{Y}, \mathbf{x})=\mathrm{p}(\mathbf{y}) \mathrm{p}(\mathbf{Y}, \mathbf{x} \mid \mathbf{y})$ and inference is based on $\mathrm{p}(\mathbf{y} \mid \mathbf{Y}, \mathbf{x})$. Let us now describe those probability distributions.

In this paper we propose a prior model based on the $\ell 1$ norm [5] to take into account the smoothness within each band plus an auto-regressive model to exploit the correlation between the image bands, thus defining the multispectral image prior

$$
\mathrm{p}(\mathbf{y}) \propto \mathrm{c}(\mathbf{y}) \prod_{b=1}^{B} \mathrm{~s}\left(\mathbf{y}_{b}\right)
$$

The term $\mathrm{c}(\mathbf{y})$, which takes into account the correlations among 
different high resolution bands, is given by

$$
c(\mathbf{y})=\exp \left\{-\sum_{\substack{b, b^{\prime}=1 \\ b^{\prime}>b}}^{B} \frac{\nu_{b b^{\prime}}}{2}\left\|\mathbf{y}_{b}-\mathbf{y}_{b^{\prime}}\right\|^{2}\right\},
$$

with $\nu_{b b^{\prime}}>0$ for $b^{\prime}>b$, thus enforcing similarity between the pixels in the same position in the different bands with $\nu_{b b^{\prime}}$ being the parameter that control the similarity between band $b$ and band $b^{\prime}$. In order to keep the flux in each band, we will normalize the bands to add to one. The smoothness into each multispectral band is modeled by the terms, $\mathrm{s}\left(\mathbf{y}_{b}\right), b=1, \ldots, B$, which are $\ell 1$ based priors defined as

$$
\mathrm{s}\left(\mathbf{y}_{b}\right)=\exp \left\{-\sum_{i=1}^{p}\left[\alpha_{b}^{h}\left\|\Delta_{i}^{h}\left(\mathbf{y}_{b}\right)\right\|_{1}+\alpha_{b}^{v}\left\|\Delta_{i}^{v}\left(\mathbf{y}_{b}\right)\right\|_{1}\right]\right\},
$$

where $\Delta_{i}^{h}\left(\mathbf{y}_{b}\right)$ and $\Delta_{i}^{v}\left(\mathbf{y}_{b}\right)$ represent the horizontal and vertical first order differences at pixel $i$, respectively, and $\alpha_{b}^{h}$ and $\alpha_{b}^{v}$ are the horizontal and vertical model parameter of the band $b$. write

We assume that $\mathbf{Y}$ and $\mathbf{x}$, for a given $\mathbf{y}$, are independent and

$$
\mathrm{p}(\mathbf{Y}, \mathbf{x} \mid \mathbf{y})=\mathrm{p}(\mathbf{Y} \mid \mathbf{y}) \mathrm{p}(\mathbf{x} \mid \mathbf{y}) .
$$

For each multispectral image band, we consider the model $\mathbf{Y}_{b}=$ $\mathbf{H y}_{b}+\mathbf{n}_{b}, b=1, \ldots, B$, where the degradation matrix $\mathbf{H}$ can be written as $\mathbf{H}=\mathbf{D B}$, with $\mathbf{B}$ a $p \times p$ blurring matrix and $\mathbf{D}$ a $P \times p$ decimation operator, and $\mathbf{n}_{b}$ is the noise term assumed to be independent white Gaussian of known variance $\beta_{b}^{-1}$. The conditional distribution of the observed image $\mathbf{Y}$ given $\mathbf{y}$ is given by

$$
\begin{aligned}
\mathrm{p}(\mathbf{Y} \mid \mathbf{y}) & =\prod_{b=1}^{B} \mathrm{p}\left(\mathbf{Y}_{b} \mid \mathbf{y}_{b}\right) \\
& \propto \prod_{b=1}^{B} \exp \left\{-\frac{1}{2} \beta_{b}\left\|\mathbf{Y}_{b}-\mathbf{H y}_{b}\right\|^{2}\right\} .
\end{aligned}
$$

The panchromatic image $\mathbf{x}$ is modeled as [3]

$$
\mathbf{x}=\sum_{b=1}^{B} \lambda_{b} \mathbf{y}_{b}+\mathbf{v}
$$

where $\lambda_{b} \geq 0, b=1,2, \ldots, B$, are known quantities that can be obtained from the sensor spectral characteristics, and $\mathbf{v}$ is the capture noise that is assumed to be Gaussian with zero mean and known variance $\gamma^{-1}$. Based on this model, the distribution of the panchromatic image $\mathbf{x}$ given $\mathbf{y}$, is given by

$$
\mathrm{p}(\mathbf{x} \mid \mathbf{y}) \propto \exp \left\{-\frac{1}{2} \gamma\left\|\mathbf{x}-\sum_{b=1}^{B} \lambda_{b} \mathbf{y}_{b}\right\|^{2}\right\}
$$

\section{BAYESIAN INFERENCE AND VARIATIONAL APPROXIMATION OF THE POSTERIOR DISTRIBUTION}

In this paper we assume that all the model parameters are known or previously estimated. Then, the Bayesian paradigm dictates that inference on $\mathbf{y}$ should be based on

$$
\mathrm{p}(\mathbf{y} \mid \mathbf{Y}, \mathbf{x})=\mathrm{p}(\mathbf{y}, \mathbf{Y}, \mathbf{x}) / \mathrm{p}(\mathbf{Y}, \mathbf{x})=\mathrm{p}(\mathbf{y}) \mathrm{p}(\mathbf{Y}, \mathbf{x} \mid \mathbf{y}) / \mathrm{p}(\mathbf{Y}, \mathbf{x}),
$$

where $\mathrm{p}(\mathbf{y}), \mathrm{p}(\mathbf{Y}, \mathbf{x} \mid \mathbf{y})$ have been defined in Eqs. (1) and (4), respectively.

Since $\mathrm{p}(\mathbf{y} \mid \mathbf{Y}, \mathbf{x})$ can not be found in closed form, we apply variational methods to approximate this distribution by a distribution $q(y)$. The variational criterion used to find $q(y)$ is the minimization of the Kullback-Leibler (KL) divergence, given by [6]

$$
\begin{aligned}
& C_{K L}(\mathrm{q}(\mathbf{y}) \| \mathrm{p}(\mathbf{y} \mid \mathbf{Y}, \mathbf{x})) \\
& \quad=\int \mathrm{q}(\mathbf{y}) \log \left(\frac{\mathrm{q}(\mathbf{y})}{\mathrm{p}(\mathbf{y} \mid \mathbf{Y}, \mathbf{x})}\right) d \mathbf{y} \\
& \quad=\int \mathrm{q}(\mathbf{y}) \log \left(\frac{\mathrm{q}(\mathbf{y})}{\mathrm{p}(\mathbf{y}, \mathbf{Y}, \mathbf{x})}\right) d \mathbf{y}+\text { const } \\
& =\mathcal{M}(\mathrm{q}(\mathbf{y}), \mathbf{Y}, \mathbf{x})+\text { const },
\end{aligned}
$$

which is always non negative and equal to zero only when $q(\mathbf{y})=$ $\mathrm{p}(\mathbf{y} \mid \mathbf{Y}, \mathbf{x})$.

Unfortunately, due to the form of the $\ell 1$ prior, the above integral can not be evaluated. However, we can rewrite $\mathrm{s}\left(\mathbf{y}_{b}\right)$ in Eq. (3) in the more convenient form

$$
\mathrm{s}\left(\mathbf{y}_{b}\right)=\exp \left\{-\sum_{i=1}^{p}\left[\alpha_{b}^{h} \sqrt{\left(\Delta_{i}^{h}\left(\mathbf{y}_{b}\right)\right)^{2}}+\alpha_{b}^{v} \sqrt{\left(\Delta_{i}^{v}\left(\mathbf{y}_{b}\right)\right)^{2}}\right]\right\}
$$

where the $\ell 1$ norm can be majorized by a function which renders the integral easier to calculate. Let us consider the following inequality, also used in [7], which states that, for any $w \geq 0$ and $z>0$

$$
\sqrt{w} \leq \frac{w+z}{2 \sqrt{z}}
$$

Let us define the functional

$$
\begin{gathered}
\operatorname{M}\left(\mathbf{y}_{b}, \mathbf{u}_{b}^{h}, \mathbf{u}_{b}^{v}\right)= \\
\exp \left\{-\sum_{i=1}^{p}\left[\frac{\alpha_{b}^{h}}{2} \frac{\left(\Delta_{i}^{h}\left(\mathbf{y}_{b}\right)\right)^{2}+\mathbf{u}_{b}^{h}(i)}{\sqrt{\mathbf{u}_{b}^{h}(i)}}+\frac{\alpha_{b}^{v}}{2} \frac{\left(\Delta_{i}^{v}\left(\mathbf{y}_{b}\right)\right)^{2}+\mathbf{u}_{b}^{v}(i)}{\sqrt{\mathbf{u}_{b}^{v}(i)}}\right]\right\},
\end{gathered}
$$

where $\mathbf{u}_{b}^{h} \in\left(R^{+}\right)^{p}, \mathbf{u}_{b}^{v} \in\left(R^{+}\right)^{p}$ are any p-dimensional vectors with components $\mathbf{u}_{b}^{h}(i)$ and $\mathbf{u}_{b}^{v}(i), i=1, \ldots, p$, that need to be computed and have, as will be shown later, an intuitive interpretation related to the unknown images $\mathbf{y}_{b}$. Using the inequality in Eq. (9) and comparing Eq. (10) with Eq. (8), we obtain $\mathrm{s}\left(\mathbf{y}_{b}\right) \geq c \cdot \mathrm{M}\left(\mathbf{y}_{b}, \mathbf{u}_{b}^{h}, \mathbf{u}_{b}^{v}\right)$. This leads to the following lower bound for the joint probability distribution

$$
\begin{aligned}
\mathrm{p}(\mathbf{y}, \mathbf{Y}, \mathbf{x}) & \geq c \cdot \mathrm{p}(\mathbf{Y} \mid \mathbf{y}) \mathrm{p}(\mathbf{x} \mid \mathbf{y}) \mathrm{c}(\mathbf{y}) \prod_{b=1}^{B} \mathrm{M}\left(\mathbf{y}_{b}, \mathbf{u}_{b}^{h}, \mathbf{u}_{b}^{v}\right) \\
& =\mathrm{F}\left(\mathbf{y}, \mathbf{Y}, \mathbf{x}, \mathbf{u}^{h}, \mathbf{u}^{v}\right)
\end{aligned}
$$

where $\mathbf{u}^{d}=\left[\mathbf{u}_{1}^{d^{t}}, \mathbf{u}_{2}^{d^{t}}, \ldots, \mathbf{u}_{B}^{d^{t}}\right]^{t}$ for $d=h, v$.

Hence, by defining

$\tilde{\mathcal{M}}\left(\mathrm{q}(\mathbf{y}), \mathbf{Y}, \mathbf{x}, \mathbf{u}^{h}, \mathbf{u}^{v}\right)=\int \mathrm{q}(\mathbf{y}) \log \left(\frac{\mathrm{q}(\mathbf{y})}{\mathrm{F}\left(\mathbf{y}, \mathbf{Y}, \mathbf{x}, \mathbf{u}^{h}, \mathbf{u}^{v}\right)}\right) d \mathbf{y}$,

and using Eq. (11), we obtain

$$
\mathcal{M}(\mathrm{q}(\mathbf{y}), \mathbf{Y}, \mathbf{x}) \leq \min _{\mathbf{u}^{h}, \mathbf{u}^{v}} \tilde{\mathcal{M}}\left(\mathrm{q}(\mathbf{y}), \mathbf{Y}, \mathbf{x}, \mathbf{u}^{h}, \mathbf{u}^{v}\right) .
$$

Therefore, by finding a sequence of distributions $\left\{q^{k}(\mathbf{y})\right\}$ that monotonically decreases $\tilde{\mathcal{M}}\left(\mathrm{q}(\mathbf{y}), \mathbf{Y}, \mathbf{x}, \mathbf{u}^{h}, \mathbf{u}^{v}\right)$ for fixed $\mathbf{u}^{h}$ and 
$\mathbf{u}^{v}$, it is also obtained a sequence of an ever decreasing upper bound of $C_{K L}(\mathrm{q}(\mathbf{y}) \| \mathrm{p}(\mathbf{y} \mid \mathbf{Y}, \mathbf{x}))$ due to Eq. (7). Even more, minimizing $\tilde{\mathcal{M}}\left(\mathrm{q}(\mathbf{y}), \mathbf{Y}, \mathbf{x}, \mathbf{u}^{h}, \mathbf{u}^{v}\right)$ with respect to $\mathbf{u}^{h}$ and $\mathbf{u}^{v}$, also generates vector sequences $\left\{\mathbf{u}^{h^{k}}\right\}$ and $\left\{\mathbf{u}^{v k}\right\}$ that tightens the upper-bound for each distribution $\mathrm{q}^{k}(\mathbf{y})$. Therefore, the sequence $\left\{\mathrm{q}^{k}(\mathbf{y})\right\}$ is coupled with the sequences $\left\{\mathbf{u}^{h^{k}}\right\}$ and $\left\{\mathbf{u}^{v k}\right\}$. We develop the following iterative algorithm to find such sequences. We note that the process to find the best posterior distribution approximation of the image in combination with $\mathbf{u}^{h}$ and $\mathbf{u}^{v}$ is a very natural extension of the Majorization-Minimization approach to function optimization [8].

\section{Algorithm 1 Posterior image distribution estimation.}

Given $\mathbf{u}^{1} \in\left(R^{+}\right)^{B p}$, for $k=1,2, \ldots$ until a stopping criterion is met:

1. Find

$$
\mathrm{q}^{k}(\mathbf{y})=\underset{\mathbf{q}(\mathbf{y})}{\arg \min _{1}} \tilde{\mathcal{M}}\left(\mathrm{q}(\mathbf{y}), \mathbf{Y}, \mathbf{x}, \mathbf{u}^{h^{k}}, \mathbf{u}^{v k}\right) .
$$

2. Find

$$
\begin{aligned}
& \left\{\mathbf{u}^{h^{k+1}}, \mathbf{u}^{v k+1}\right\}= \\
& \quad \operatorname{argmin}_{\left\{\mathbf{u}^{h}, \mathbf{u}^{v}\right\}} \tilde{\mathcal{M}}\left(\mathbf{q}^{k}(\mathbf{y}), \mathbf{Y}, \mathbf{x}, \mathbf{u}^{h}, \mathbf{u}^{v}\right) .
\end{aligned}
$$

$\operatorname{Set} \mathrm{q}(\mathbf{y})=\lim _{k \rightarrow \infty} \mathrm{q}^{k}(\mathbf{y})$.

To calculate $\left\{\mathbf{u}^{h^{k+1}}, \mathbf{u}^{v k+1}\right\}$, for $b=1, \ldots, B$, we have from Eq. (13) that

$$
\mathbf{u}_{b}^{h^{k+1}}=\arg \min _{\mathbf{u}_{b}^{h}} \sum_{i=1}^{P} \frac{\mathbf{E}_{\mathbf{q}^{k}(\mathbf{y})}\left[\left(\Delta_{i}^{h}\left(\mathbf{y}_{b}\right)\right)^{2}\right]+\mathbf{u}_{b}^{h}(i)}{\sqrt{\mathbf{u}_{b}^{h}(i)}},
$$

and

$$
\mathbf{u}_{b}^{v k+1}=\arg \min _{\mathbf{u}_{b}^{v}} \sum_{i=1}^{P} \frac{\mathbf{E}_{\mathrm{q}^{k}(\mathbf{y})}\left[\left(\Delta_{i}^{v}\left(\mathbf{y}_{b}\right)\right)^{2}\right]+\mathbf{u}_{b}^{v}(i)}{\sqrt{\mathbf{u}_{b}^{v}(i)}},
$$

and consequently

$$
\left.\mathbf{u}_{b}^{h^{k+1}}(i)=\mathbf{E}_{\mathbf{q}^{k}(\mathbf{y})}\left[\Delta_{i}^{h}\left(\mathbf{y}_{b}\right)\right)^{2}\right],
$$

and

$$
\left.\mathbf{u}_{b}^{v k+1}(i)=\mathbf{E}_{\mathbf{q}^{k}(\mathbf{y})}\left[\Delta_{i}^{v}\left(\mathbf{y}_{b}\right)\right)^{2}\right],
$$

for $i=1, \ldots, p$ and $b=1, \ldots, B$.

It is clear from Eqs. (14) and (15) that vectors $\mathbf{u}_{b}^{h^{k+1}}$ and $\mathbf{u}_{b}^{v k+1}$ are respectively functions of the spatial first order horizontal and vertical differences of the unknown image $y$ under the distribution $\mathrm{q}^{k}(\mathbf{y})$ and represent the local spatial activity of $\mathbf{y}$.

To calculate $\mathrm{q}^{k}(\mathbf{y})$, we observe that differentiating the integral on the right-hand side of Eq. (12) with respect to $q(\mathbf{y})$ and setting it equal to zero, we obtain that

$$
\mathrm{q}^{k}(\mathbf{y})=\mathcal{N}\left(\mathbf{y} \mid \mathbf{E}_{q^{k}(\mathbf{y})}[\mathbf{y}], \operatorname{cov}_{q^{k}(\mathbf{y})}[\mathbf{y}]\right),
$$

with

and

$$
\operatorname{cov}_{q^{k}(\mathbf{y})}[\mathbf{y}]=\mathcal{A}^{-1}\left(\mathbf{u}^{h^{k}}, \mathbf{u}^{v k}\right)
$$

$$
\mathbf{E}_{q^{k}(\mathbf{y})}[\mathbf{y}]=\operatorname{cov}_{q^{k}(\mathbf{y})}[\mathbf{y}] \phi^{k}
$$

where $\phi^{k}$ is the $(B \times p) \times 1$ vector

$$
\phi^{k}=\left(\operatorname{diag}(\beta) \otimes \mathbf{H}^{t}\right) \mathbf{Y}+\gamma(\lambda \otimes \mathbf{x}),
$$

and

$$
\begin{gathered}
\mathcal{A}\left(\mathbf{u}^{h^{k}}, \mathbf{u}^{v k}\right)=\operatorname{diag}(\beta) \otimes \mathbf{H}^{t} \mathbf{H}+\gamma\left(\lambda \lambda^{t}\right) \otimes \mathbf{I}_{p} \\
+\left(\begin{array}{cccc}
\mathcal{G}\left(\mathbf{u}_{1}^{h}, \mathbf{u}_{1}^{v k}\right) & \mathbf{0}_{p} & \ldots & \mathbf{0}_{p} \\
\mathbf{0}_{p} & \mathcal{G}\left(\mathbf{u}_{2}^{h^{k}}, \mathbf{u}_{2}^{v k}\right) & \ldots & \mathbf{0}_{p} \\
\vdots & \vdots & \ddots & \vdots \\
\mathbf{0}_{p} & \mathbf{0}_{p} & \ldots & \mathcal{G}\left(\mathbf{u}_{B}^{h^{k}}, \mathbf{u}_{B}^{v}{ }^{k}\right)
\end{array}\right) \\
+\left(\begin{array}{cccc}
\sum_{b=1}^{B} \mu_{1 b} & -\mu_{12} & \ldots & -\mu_{1 B} \\
-\mu_{21} & \sum_{b=1}^{B} \mu_{2 b} & \ldots & -\mu_{2 B} \\
\vdots & \vdots & \ddots & \vdots \\
-\mu_{B 1} & -\mu_{B 2} & \ldots & \sum_{b=1}^{B} \mu_{B b}
\end{array}\right) \otimes \mathbf{I}_{p},
\end{gathered}
$$

where $\otimes$ is the Kronecker product, $\beta=\left(\beta_{1}, \beta_{2}, \ldots, \beta_{B}\right)^{t}, \lambda=$ $\left(\lambda_{1}, \lambda_{2}, \ldots, \lambda_{B}\right)^{t}, \mu_{b b}=0, \forall b, \mu_{b b^{\prime}}=\nu_{b^{\prime} b}, b^{\prime}>b$ and $\mu_{b^{\prime} b}=$ $\mu_{b b^{\prime}}$

$$
\mathcal{G}\left(\mathbf{u}_{b}^{h^{k}}, \mathbf{u}_{b}^{v k}\right)=\alpha_{b}^{h} \Delta^{h^{t}} W\left(\mathbf{u}_{b}^{h^{k}}\right) \Delta^{h}+\alpha_{b}^{v} \Delta^{v t} W\left(\mathbf{u}_{b}^{v k}\right) \Delta^{v},
$$

for $b=1, \ldots, B$, where $\Delta^{h}$ and $\Delta^{v}$ represent $p \times p$ convolution matrices associated with the first order horizontal and vertical differences, respectively, and $W\left(\mathbf{u}_{b}^{h^{k}}\right)$ and $W\left(\mathbf{u}_{b}^{v k}\right)$ are a $p \times p$ diagonal matrices of the form $W\left(\mathbf{u}_{b}^{d^{k}}\right)=\operatorname{diag}\left(\mathbf{u}_{b}^{d^{k}}(i)^{-\frac{1}{2}}\right)$, for $i=1, \ldots, p, d=h, v$. These matrices can be interpreted as spatial adaptivity matrices since they controls the amount of smoothing at each pixel location depending on the strength of the intensity variation at that pixel, as expressed by the horizontal and vertical intensity gradients, respectively. That is, for pixels with high spatial activity in the horizontal (vertical) direction, the corresponding entries of $W\left(\mathbf{u}_{b}^{h^{k}}\right)\left(W\left(\mathbf{u}_{b}^{v k}\right)\right)$ are very small, which means that no smoothness is enforced in this direction, while for areas where pixels have similar values in the horizontal or vertical direction the corresponding entries of $W\left(\mathbf{u}_{b}^{h^{k}}\right)$ or $W\left(\mathbf{u}_{b}^{v k}\right)$, respectively, are very large, which means that smoothness is enforced in the corresponding direction.

\section{EXPERIMENTAL RESULTS}

Although we performed a wide set of experiments to assess the quality of the proposed approach, here we report results only on a synthetic color image and a real Landsat multispectral image.

Our first experiment was conducted on the $120 \times 160$ color image displayed in Fig. 1(a) and helps to illustrate the usefulness of the term that takes into account the correlation between image bands (see Eq. (2)) in the prior model. To obtain a low resolution color image, each band of the original image was first convolved with the mask $0.25 \times \mathbf{1}_{2 \times 2}$ to simulate sensor integration, and then downsampled by a factor of two by discarding every other pixel in each direction. Zero mean Gaussian noise was finally added to each band to obtain two observed multispectral images with a signal-to-noise ratio (SNR) of $20 \mathrm{~dB}$ and 30 $\mathrm{dB}$, each. The panchromatic image was obtained from the original high resolution color image using the model in Eq. (6), with $\lambda=[0.3,0.6,0.1]^{t}$, and adding zero mean Gaussian noise with 


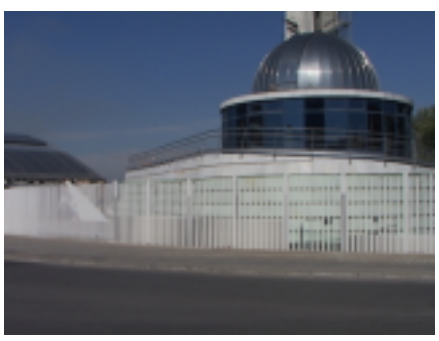

(a)

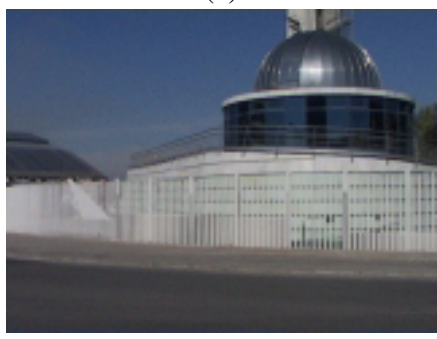

(d)

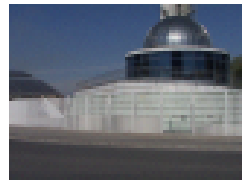

(b)

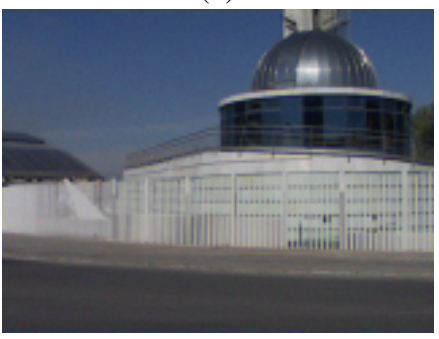

(e)

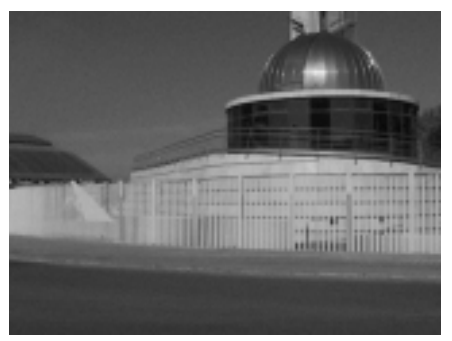

(c)

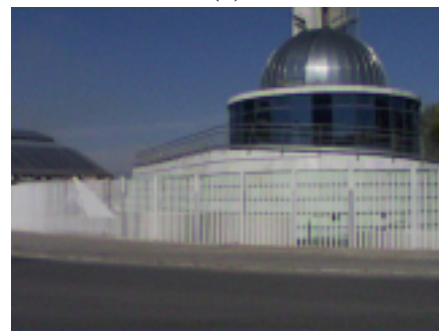

(f)

Fig. 1. (a) Original RGB image; (b) Corresponding synthetic multi-spectral image and (c) synthetic panchromatic image; (d) Reconstruction using the proposed method; (e) Reconstruction using $\ell 1$ method; (f) Reconstruction using the method in [3].

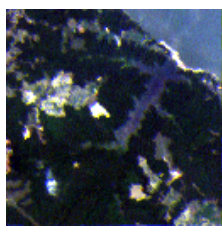

(a)

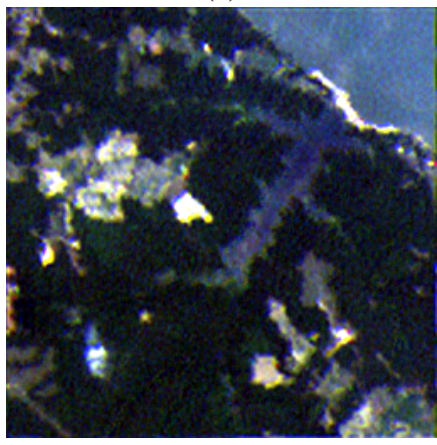

(d)

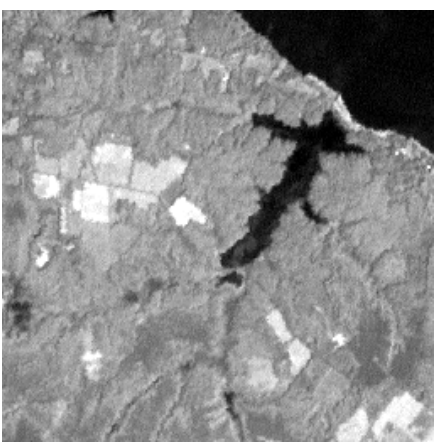

(b)

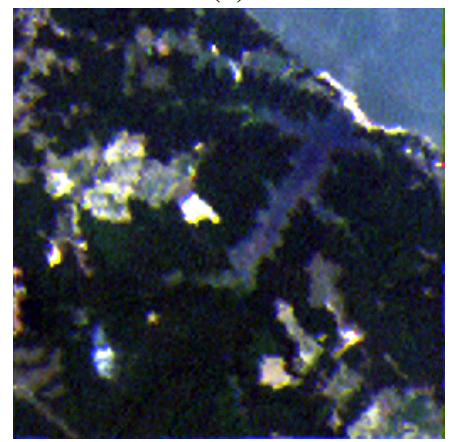

(e)

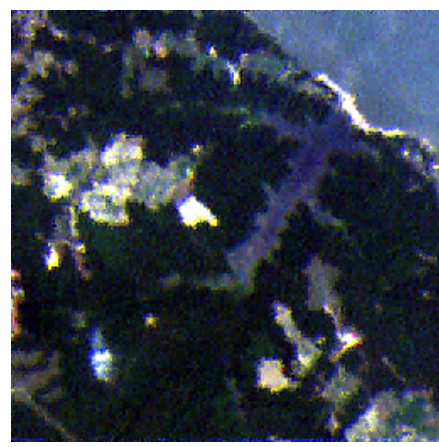

(c)

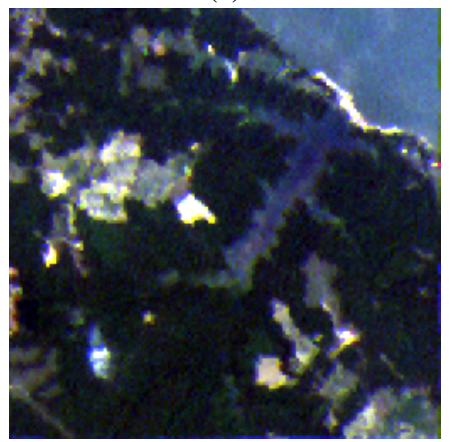

(f)

Fig. 2. (a) Region of interest of the observed multispectral image; (b) Corresponding region of interest of the observed panchromatic image; (c) Reconstruction using the method in [2]; (d) Reconstruction using the method in [3]; (e) Reconstruction using the method in [12]; (f) Reconstruction using the proposed method. 
Table 1. Values of PSNR, COR, SSIM and ERGAS values for the synthetic color image.

\begin{tabular}{c|l|ccc|cccc|ccc|c}
\hline & & \multicolumn{4}{c}{ PSNR } & \multicolumn{4}{c}{ COR } & \multicolumn{4}{c}{ SSIM } & \multicolumn{1}{c}{ ERGAS } \\
\hline SNR & Method / Band & 1 & 2 & 3 & 1 & 2 & 3 & 1 & 2 & 3 & \\
\hline \multirow{3}{*}{$20 \mathrm{~dB}$} & Bicubic interpolation & 25.5 & 25.7 & 25.6 & 0.37 & 0.38 & 0.37 & 0.67 & 0.68 & 0.68 & 6.88 \\
& method in [3] & 32.8 & 35.6 & 29.6 & 0.97 & 0.90 & 0.98 & 0.63 & 0.97 & 0.90 & 3.19 \\
& $\ell 1$ method & 32.9 & 34.2 & 30.5 & 0.98 & 0.99 & 0.77 & 0.83 & 0.86 & 0.84 & 3.12 \\
& proposed method & 33.2 & 34.5 & 33.1 & 0.97 & 0.99 & 0.95 & 0.84 & 0.87 & 0.86 & 2.74 \\
\hline \multirow{3}{*}{$30 \mathrm{~dB}$} & Bicubic interpolation & 29.2 & 29.2 & 29.3 & 0.53 & 0.54 & 0.52 & 0.84 & 0.85 & 0.85 & 4.50 \\
& method in [3] & 36.4 & 40.9 & 30.6 & 0.96 & 0.99 & 0.71 & 0.96 & 0.98 & 0.97 & 2.45 \\
& $\ell 1$ method & 40.1 & 42.4 & 31.9 & 0.99 & 1.00 & 0.79 & 0.97 & 0.98 & 0.90 & 2.00 \\
& proposed method & 40.3 & 42.8 & 37.7 & 0.99 & 1.00 & 0.97 & 0.97 & 0.98 & 0.95 & 1.31 \\
\hline
\end{tabular}

Table 2. Values of PSNR, COR and ERGAS for the Landsat image in Fig. 2.

\begin{tabular}{l|cccc|ccccc|c}
\hline & \multicolumn{9}{|c}{ PSNR } & \multicolumn{7}{c}{ COR } & ERGAS \\
\hline Method / Band & 1 & 2 & 3 & 4 & 1 & 2 & 3 & 4 & \\
\hline method in [2] & 39.8 & 39.6 & 35.5 & 35.6 & 0.73 & 0.75 & 0.62 & 0.91 & 2.75 \\
method in [3] & 42.5 & 41.9 & 37.5 & 29.1 & 0.70 & 0.78 & 0.84 & 0.91 & 3.20 \\
method in [12] & 42.2 & 42.0 & 37.5 & 28.8 & 0.69 & 0.76 & 0.77 & 0.96 & 3.29 \\
proposed method & 42.3 & 41.9 & 37.6 & 29.3 & 0.69 & 0.75 & 0.79 & 0.93 & 3.15 \\
\hline
\end{tabular}

an SNR of $20 \mathrm{~dB}$ and $30 \mathrm{~dB}$. Details of the $30 \mathrm{~dB}$ observed multispectral and panchromatic images are depicted in Figs. 1(b) and $1(\mathrm{c})$, respectively.

To assess the quality of multispectral images both spatial and spectral measures have to be considered. Spatial improvement was assessed by means of the correlation of the high frequency components (COR) [9] which takes values between 0 and 1 (the higher the value the better the quality of the reconstruction), while spectral fidelity was assessed by means of the peak signal-to-noise ratio (PSNR) and SSIM index [10] between each band of the reconstructed and original multispectral images, and the standard ERGAS index [11] for which the lower the value, especially a value under the number of bands $B$, the higher the quality of the multispectral reconstructed image.

We run the proposed algorithm until the criterion $\| \mathbf{E}_{q^{k}(\mathbf{y})}[\mathbf{y}]-$ $\mathbf{E}_{q^{k-1}(\mathbf{y})}[\mathbf{y}]\left\|^{2} /\right\| \mathbf{E}_{q^{k-1}(\mathbf{y})}[\mathbf{y}] \|^{2}<10^{-4}$ was satisfied. The values of the parameters were determined experimentally using a method similar to the one presented in [3]. The obtained reconstruction is shown in Fig. 1(d). To illustrate the usefulness of the proposed approach we show the reconstructed image obtained by the proposed method without considering the correlation between the bands (henceforth $\ell 1$ method), that is, using the proposed method with $\nu_{b, b^{\prime}}=0, \forall b, b^{\prime}=1, \ldots, B$ (see Fig. 1(e)). The reconstruction obtained using the method in [3] which utilizes a simultaneous auto-regressive prior model in shown in Fig. 1(f)). From the displayed images it is clear that the proposed method results in a better reconstruction. The new method obtains sharper edges than the method in [3] and achieves higher similarity to the colors of the original image.

Numerical results, presented in Table 1, also support the use of the proposed model. Its figures of merit are, in general, better than the ones provided by all other methods, highly increasing the PSNR and the COR coefficient for band 3 except for the band 3 of the $20 \mathrm{~dB}$ SNR image where the COR values are similar to the ones obtained by the method in [3]. It also obtains a larger SSIM value than the $\ell 1$ method for band 3 thus obtaining a more consistent quality for all the bands. The table also includes the figures of merits of the reconstructions by bicubic interpolation as a reference. The quality improvement of the proposed method clearly manifests itself when the ERGAS index is examined, since the value is highly reduced when the proposed method is utilized.

In a second experiment, the method was tested on a real Landsat ETM+ image. Figure 2(a) depicts a $128 \times 128$ RGB color region of interest composed of bands 3,2 , and 1 of a Landsat ETM+ multispectral image, and Fig. 2(b) depicts its corresponding $256 \times 256$ panchromatic image region. According to the ETM+ sensor spectral response, the panchromatic image covers only the spectrum of a part of the first four bands of the multispectral image. Hence, we apply the proposed method with $B=4$. The values of $\lambda_{b}, b=1,2,3,4$, calculated from the spectral response of the ETM+ sensor, are equal to $0.0078,0.2420,0.2239$, and 0.5263 , for bands one to four, respectively [3]

Note that in this experiment we do not have access to the original high resolution multi-spectral image to numerically evaluate the quality of the different reconstructions. In order to obtain a numerical comparison, like the one in the first experiment, and to overcome the lack of an exact HR multi-spectral image to compare with, an intermediate step has been performed in which a simulated multispectral image of size $64 \times 64$ pixels and its corresponding panchromatic image of size $128 \times 128$ pixels have been obtained from the observed $128 \times 128$ pixels multispectral image and its corresponding $256 \times 256$ pixels panchromatic image by applying a simulated observation process, that is, applying the sensor integration and downsampling the observed image. A numerical comparison between the different $128 \times 128$ reconstructions and the observed $128 \times 128$ multispectral image is presented in $\mathrm{Ta}-$ ble 2. The table includes, together with the proposed method, numerical results for the pansharpening method proposed in [2], the method proposed in [3] and the method proposed in [12] which uses a total variation (TV) prior model. The corresponding reconstructed images are displayed in Fig. 2. A visual inspection of the images shows that the method in [2] (Fig. 2(c)) produces sharp but very noisy reconstructions, creating artifacts at the boundaries of the objects due to noise. The method in [3] (Fig. 2(d)) produces smooth reconstructions, smoothing out sharp edges but controls the noise. Note however that colors are not very well recon- 
structed due to the poor contribution of the panchromatic image to band 1. The method in [12] (Fig. 2(e)) has crisper edges and good noise control but, as the method in [3], does not reconstruct well the colors. However, the proposed method (Fig. 2(f)) successfully includes the high frequencies of the panchromatic image, producing sharp edges and maintaining the small details. Furthermore, it also preserves the spectral quality of the image by including in the model the correlation among the bands.

\section{CONCLUSIONS}

We have presented a new method for pansharpening of multispectral images using a super resolution approach which takes into account the sensor characteristics in the image formation model and incorporates prior knowledge on the expected characteristics of multispectral images by imposing smoothness within each band and by taking into account the correlation between the bands of the multispectral image. This correlation is, to the best of our knowledge, the first time that it is explicitly used in pansharpening. We have used the variational approach to approximate the posterior distribution of the pansharpened multispectral image. Based on the presented experimental results, the proposed method successfully incorporates the high frequencies of the panchromatic image into the reconstructed image while preserving the spectral quality of the image.

\section{REFERENCES}

[1] J. Nuñez, X. Otazu, O. Fors, A. Prades, V. Pala, and R. Arbiol, "Multiresolution-based image fusion with additive wavelet decomposition," IEEE Trans on Geosc. \& Rem. Sens., vol. 37, no. 3, pp. 1204-1211, 1999.

[2] J.C. Price, "Combining multispectral data of different spatial resolution," IEEE Trans. on Geosc. \& Rem. Sens., vol. 37, no. 3, pp. 1199-1203, 1999.

[3] R. Molina, M. Vega, J. Mateos, and A.K. Katsaggelos, "Variational posterior distribution approximation in Bayesian super resolution reconstruction of multispectral images," Applied and Computational Harmonic Analysis, vol. 24, no. 2, pp. 251-267, 2008.

[4] L. Alparone, L. Wald, J. Chanussot, C. Thomas, P. Gamba, and L.M. Bruce, "Comparison of pansharpening algorithms: Outcome of the 2006 GRS-S data-fusion contest," IEEE Trans. on Geosc. \& Rem. Sens., vol. 45, no. 10, pp. 30123020, 2007.

[5] M. Vega, R. Molina, and A.K. Katsaggelos, "L1 prior majorization in Bayesian image restoration," in 16th Int. Conf. on Digital Signal Processing DSP 2009, 2009.

[6] S. Kullback and R. A. Leibler, "On information and sufficiency," Annals of Math. Stat., vol. 22, pp. 79-86, 1951.

[7] J. Bioucas-Dias, M. Figueiredo, and J. Oliveira, "Total-variation image deconvolution: A majorizationminimization approach,' in ICASSP'2006, 2006.

[8] K. Lange, "Optimization," in Springer texts in Statistic. New York, Springer Verlag, 2004.

[9] V. Vijayaraj, "A quantitative analysis of pansharpened images,” M.S. thesis, Mississippi St. University, 2004.
[10] Z. Wang, A. C. Bovik, H. R. Sheikh, and E. P. Simoncelli, "Image quality assessment: From error visibility to structural similarity," IEEE Trans. on Img Proc., vol. 13, no. 4, pp. 600-612, 2004.

[11] L. Wald, T. Ranchin, and M. Mangolini, "Fusion of satellite images of different spatial resolutions: assessing the quality of resulting images," Phot. Eng. Rem. Sens., vol. 63, no. 6, pp. 691-699, 1997.

[12] M. Vega, J. Mateos, R. Molina, and A.K. Katsaggelos, "Super resolution of multispectral images using TV image models," in 2th Int. Conf. on Knowledge-Based and Intelligent Information \& Engineering Systems, 2008, pp. 408-415. 